\section{Ageing of Bone Structure and the Risk of Osteoporosis in the Menopausal Transition

\author{
Annamaria Zsakai ${ }^{1 *}$, Nicholas Mascie-Taylor ${ }^{2}$ and Eva B \\ Bodzsar $^{1}$
}

\begin{abstract}
Purpose: The purpose of the research was to study the relationship between menopausal status and bone structure during the menopausal transition.

Methods: A random sample of 1932 Hungarian women was enrolled in the study. Bone mass was estimated by the Drinkwater-Ross method. Bone structure parameters were assessed by a quantitative ultrasound (QUS) device. High and very high risk of osteoporosis was identified by using the thresholds of QUS parameters.

Results: By considering the changes in QUS parameters and bone mass by age and reproductive age an intensive, menopause-related change from the late $40 \mathrm{~s}$ and then another significant change from the beginning of the 70s were observed in bone. The bone mass decreased while the porosity of the bone component of the female body decreased by age and by menopausal status. On average 15$17 \%$ of women are at very high risk of developing osteoporosis in the premenopausal status and after the menopausal transition the decreased level of female sex hormone production doubles this risk of osteoporosis from the beginning of the postreproductive period, triples this risk for women in the seventies.
\end{abstract}

Conclusion: The results emphasize the importance of menopausal status assessment in screening for age-related increase risk of osteoporosis.

\section{Keywords}

Risk of osteoporosis; Quantitative ultrasound parameters; Bone structure; Bone mass; Menopausal transition

\section{Introduction}

Osteoporosis, one of the most frequent skeletal disorders, is the imbalance of the bone remodelling when the balance of the bone resorption and formation functions is disturbed. The remodelling of bones is influenced by many internal and external factors: it has a strong genetic component, it is strictly regulated by the endocrine system, but it is also an age-, diet-, activity-, drug consumption-related disorder [1]. Osteoporosis is characterized by low bone mass and structural deterioration of the trabecular bone microarchitecture, and the structural changes arising from physiological and biomechanical processes result in reduced bone strength, mechanical weakness and increased fracture risk [2].

*Corresponding author: Annamaria Zsakai, Department of Biological Anthropology, Eotvos Lorand University, Pazmany P. s. 1/c, 1117 Budapest, Hungary, Tel: +36 1381 2161; E-mail: zsakaia@elte.hu

Received: March 15, 2017 Accepted: April 13, 2017 Published: April 18, 2017
Osteoporosis has reached epidemic proportions worldwide and 1 in 3 women and 1 in 5 men over age 50 experience osteoporotic fractures [3-6]. The ageing of the populations in developed countries, the clinical consequences and economic burden of this disease emphasize the importance of the population-wide screening programmes of the disease to identify the most important risk factors of osteoporosis and its pre diagnostic forms as early as possible.

The diagnosis of osteoporosis can be made by the examination of bone density and bone quality. Bone density tends to be assessed by medical imaging techniques on different regions of the skeleton, while bone quality is usually assessed on the basis of the subject's history of a fragility fracture. In spite of its potential for widespread use at present it is used in the screening but not in the diagnosis of osteoporosis contrary to the "gold standard" central densitometry techniques. Since the diagnostic sensitivity of quantitative ultrasound (QUS) method has been described as sensitive as in the dual $\mathrm{x}$-ray absorptiometry (DEXA) in the last decades [7-9] and QUS is radiation free, portable, developed for scanning the peripheral regions of the skeleton and inexpensive it is considered nowadays as an alternative to photon absorptiometric methods in the skeletal status assessment in osteoporosis. Moreover, both the structure of the bones and on the basis of structural information bone mass density can be assessed by the QUS method.

The WHO Collaborating Centre for Metabolic Bone Diseases $[10,11]$ recommended the $\mathrm{T}$ score $\leq-2.5$ threshold of bone mineral density to diagnose osteoporosis in photon absorptiometric methods. However, the combined analyses of $\mathrm{x}$-ray and ultrasonic methods revealed this WHO criteria of osteoporosis cannot be used for QUS methods [9]. Therefore thresholds for several QUS peripheral devices for osteoporosis risk assessment were suggested [12] by analysing the QUS parameters of subjects with and without total hip DEXA osteoporosis diagnosis.

Women from their late 40s are at higher risk of developing osteoporosis due to the body and bone structural changes (higher risk of obesity, low bone mass and bone mineral density) caused by the low levels of endogenous sex hormones, the decreased level of physical activity and the metabolic changes of the menopausal transition [13-18]. The main purposes of the study were (1) to study the bone status of women in the menopausal transition, and (2) to assess the risk of osteoporosis in the menopausal transition by a QUS method. The bone status of subjects were characterized by bone mass, BUA and SOS QUS parameters, as well as by the stiffness index derived from the QUS parameters. The DTU-one osteometer, the imaging ultrasound scanner used in the study, examines the structure of the calcaneus bone. The DTU-one device was used since the very first sign of the forming osteoporotic structure in the skeleton can usually be observed in the calcaneus bone, since it is greater than $90 \%$ trabecular volume, and there is little overlying tissue in this region of interest.

\section{Subjects and Methods}

\section{Sample}

After body structural (anthropometric, body composition and osteometric) examinations women (n: 1932, aged 35+ years; (Table 
1)) were interviewed by questionnaires concerning their reproductive and menstrual history, socio-demographic background, lifestyle, health conditions and subjective health during a cross-sectional survey between 2011 and 2014 [19]. Women who had any diseases or were taking any medications known to affect body composition, or who were hysterectomized or ovariectomized, were not included in the analysis. Sample selection was done by considering the recommendations of the Sampling and Methodology Section of the Hungarian Central Statistical Office, which were based on the population statistics of the administrative territories and designstatistical regions.

Subjects were divided into ten-year age-groups (Table 1). Subjects were also divided into menopausal status (premenopausal, early and late perimenopausal and postmenopausal) subgroups on the basis of the occurrence of irregular periods and the age of last menstrual period. Only the joint premenopausal (women with menstrual bleeding in the past 12 months) and the postmenopausal (women who were amenorrheic for the past 12 months and no hysterectomy) subgroups were used in the present analysis. Premature menopause was diagnosed if the onset of menopause occurred before age 40 .

\section{Data collection}

Bone mass was estimated by the Drinkwater-Ross [20] anthropometric four-component method. Bone structure was examined by a DTU-one ultrasound device (Osteometer Meditech, California, USA), two ultrasound parameters on the left calcaneus bone were measured: broadband ultrasound attenuation (BUA, $\mathrm{dB} / \mathrm{MHz}$ ) and speed of sound (SOS, $\mathrm{m} / \mathrm{s}$ ). An additional ultrasonic parameter, the stiffness index $(\mathrm{SI}=0.67 \times \mathrm{BUA}+0.28 \times \mathrm{SOS}-420)$ [21] was derived from the individual BUA and SOS parameters to minimize the measurement errors caused by the variation of heel width and water temperature $[22,23]$.

The level of risk for osteoporosis was estimated by using the QUS parameters' thresholds (BUA $\leq 35.1 \mathrm{~dB} / \mathrm{MHz}, \mathrm{SOS} \leq 1528 \mathrm{~m} / \mathrm{s}$ ) introduced by [12] for the DTU-one quantitative ultrasonic device of peripheral bone status assessment. The thresholds were constructed in women aged between 55-80 years for the DTU-one device that identified women with osteoporosis with the same specificity and sensitivity as total hip DXA at $95 \%$ confidence level (95\% certainty that an individual had osteoporosis). The threshold values of both BUA and SOS were used in the present analysis. Women having lower level of one QUS parameter than the threshold were considered being at high risk of osteoporosis, while those women whose both QUS parameters were lower than the thresholds were identified being at very high risk of osteoporosis

\section{Data management and statistical analysis}

The computations and the statistical analyses were carried out using SPSS v. 20. Hypotheses were tested at the 5\% level of random error. The centile curves of bone structural parameters were estimated by using lmsChartMaker Pro 2.3 software $[24,25]$ based on the LMS method.

Informed consent was obtained from all individual participants included in the study. All participants were provided with detailed information on the main purposes of the study and on all examinations before their approval. The participation was voluntary and anonymous. The research objectives, the research methodology and the questionnaires were approved by the National Human Research Ethics Committee (108/2011) and the Hungarian Scientific
Research Fund (EIK-1001/2011). All procedures performed in studies involving human participants were in accordance with the ethical standards of the institutional and/or national research committee and with the 1964 Helsinki declaration and its later amendments or comparable ethical standards.

\section{Results}

Since international or foreign references are used in the Hungarian screening surveys of skeletal status, the reference quantitative ultrasound data in the studied age-group of Hungarian women were constructed for the DTU-one sonometer. By considering the reference centiles it could be stated, that all the QUS parameters decreased by age in women in the studied age interval, however an accelerated decrease was observed in SOS from the age of 45 (Figure 1). The absolute bone mass was found to be stable until the age of 65 , and then revealed a decrease until the end of the studied age interval. There was a tendency for premenopausal women have a higher QUS parameters than postmenopausal age-peers, but the bone mass of women of differing menopausal status of the same age did not vary in the studied sample (Figure 1).

The risk of osteoporosis increased by age in the postmenopausal subgroup, however very intensively after the age of 75 years (Figure 2). The risk of osteoporosis did not change by age in the premenopausal subgroup, the prevalence of women with high level osteoporosis ranged between $1.5-2.5 \%$, while the prevalence of women with very high level osteoporosis ranged between $14.5-17.0 \%$ in women in the reproductive period. This means generally $15-17 \%$ of women are at very high risk for osteoporosis, and after the menopausal transition the decreased level of female sex hormone production doubles this risk for the beginning of the post reproductive period, and triples this risk for women in their 70s (Figure 2).

The pattern of BUA and SOS parameters in women who experienced premature menopause was different from the general centile distribution of QUS parameters. Both BUA and SOS parameters of women with premature menopause were significantly smaller than women in their age-groups (Figure 3).

\section{Discussion}

The potential of using a quantitative ultrasound method in the screening for osteoporosis has not been fully utilized. However, the International Quantitative Ultrasound Consensus Group has already suggested the method for fracture risk prediction at least in elderly women [26,27]. On the basis of their recommendation an assessment of women's skeletal status in the menopausal transition was carried out to study the ageing processes of bone mass and structure and to evaluate the osteoporotic risk by the reproductive ageing.

By considering the results of the present analysis the following could be concluded:

Table 1: The distribution of subjects by age-groups.

\begin{tabular}{|l|l|l|}
\hline Age-group (years) & $\mathbf{n}$ & $\%$ \\
\hline $25.1-35.0$ (Age-group 30) & 185 & 9.6 \\
\hline $35.1-45.0$ (Age-group 40) & 192 & 9.9 \\
\hline $45.1-55.0$ (Age-group 50) & 256 & 13.3 \\
\hline $55.1-65.0$ (Age-group 60) & 357 & 18.5 \\
\hline $65.1-75.0$ (Age-group 70) & 275 & 14.2 \\
\hline $75.1-85.0$ (Age-group 80) & 347 & 18.0 \\
\hline 85.1-95.0 (Age-group 90) & 320 & 16.6 \\
\hline Together & 1932 & 100.0 \\
\hline
\end{tabular}


Citation: Zsakai A, Mascie-Taylor N, Bodzsar EB (2017) Ageing of Bone Structure and the Risk of Osteoporosis in the Menopausal Transition. J Womens Health, Issues Care 6:3.
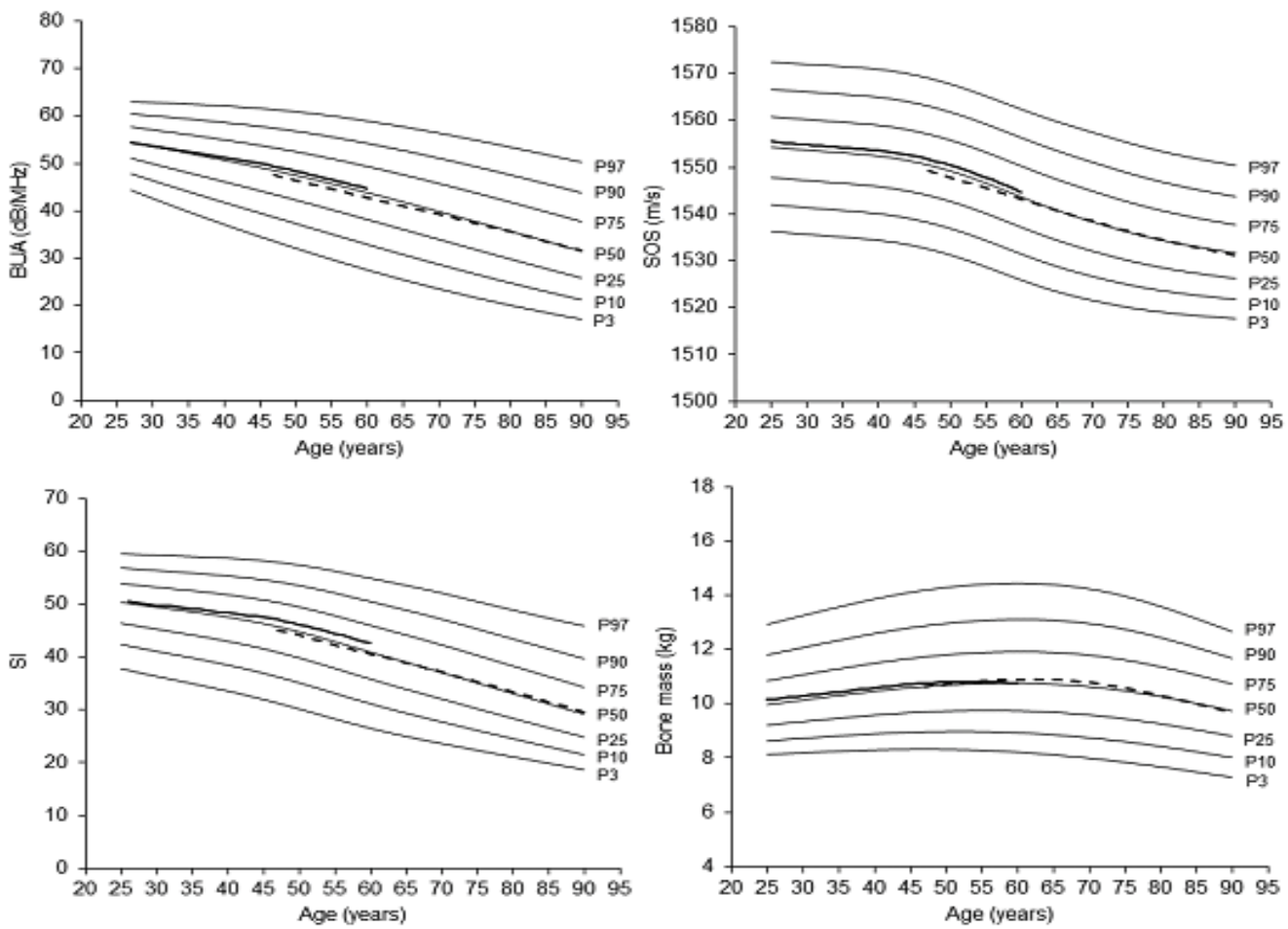

Figure 1: The centile distribution of quantitative ultrasound parameters (calcaneus, DTU-one) and absolute bone mass in women aged between 25 and 90 years (median curves of premenopausal: — and postmenopausal: - - - subgroups).

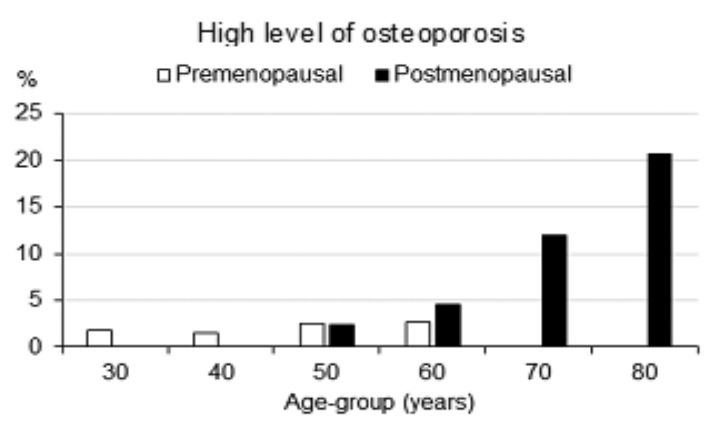

Ver y high level of osteo porosis

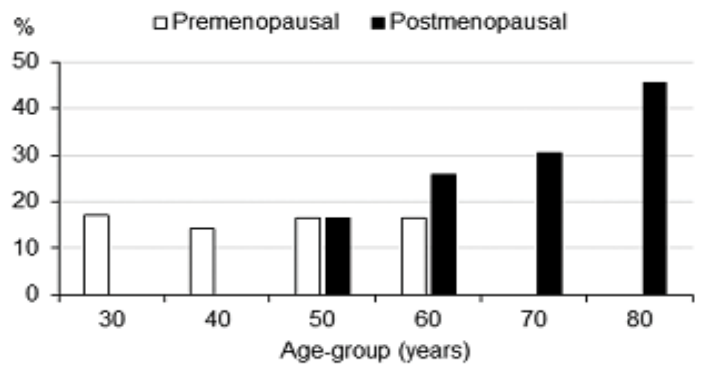

Figure 2: Prevalence (\%) of women identified with high and very high level of osteoporosis by menopausal status.

(1) The changes in the sex hormone levels in the menopausal transition manifested in the structural parameters of the bones but not in the absolute amount of bone mass.

(2) The risk of osteoporosis in women did not change until the
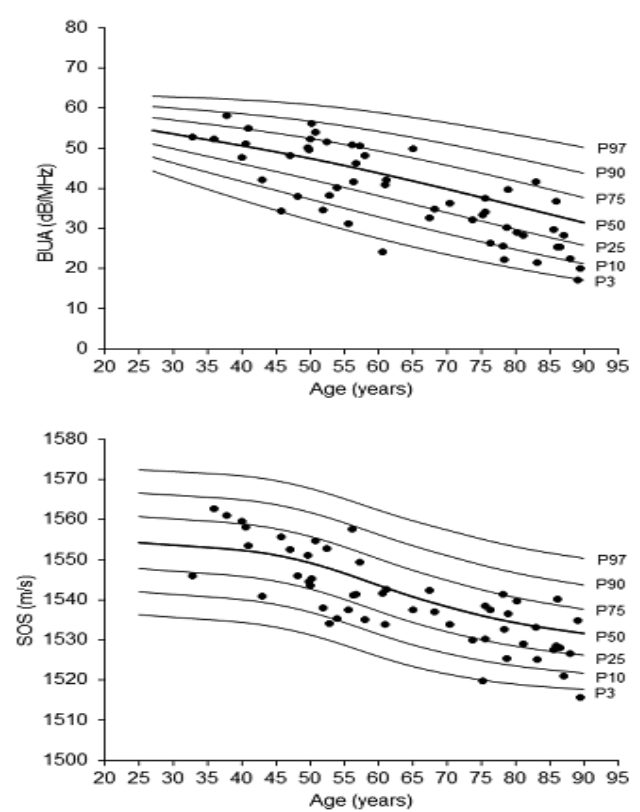

Figure 3: BUA and SOS quantitative ultrasound parameters of women $(\bullet)$ with premature menopause (age at menopause $\leq 45$ years) in the centile distribution of the studied population' QUS parameters (BUA and SOS: $\mathrm{p}<0.05, \mathrm{x}^{2}$ test)

beginning of the post reproductive period, and ranged between 15$17 \%$, while the risk doubled in the menopausal transition and there was another shift towards an increased risk from the age 70 .

(3) Premature menopause indicates that the hormonal status in 
Citation: Zsakai A, Mascie-Taylor N, Bodzsar EB (2017) Ageing of Bone Structure and the Risk of Osteoporosis in the Menopausal Transition. J Womens Health, Issues Care 6:3.

the reproductive period predicts the bone status not only at the end of the reproductive period but through the whole post reproductive period.

Limitation of the study was the design of methodology. The level of risk for osteoporosis was estimated by using the international thresholds of QUS parameters. The thresholds were constructed only for women aged between 55-80 years, and the thresholds are not age-dependent cut-off values [12]. The methodology of menopausal status estimation also impacted the results of study, since only the occurrence of irregular periods and the age of last menstrual period were, but sex hormonal status was not considered. However, the results of the analysis emphasize the importance of menopausal status assessment in screening for age-related increase risk of osteoporosis.

\section{Acknowledgements}

The study was supported by the Hungarian Scientific Research Fund unde OTKA grant K83966. The work of the first author was also supported by the Janos Bolyai Research Scholarship (2014-2017) of the Hungarian Academy of Sciences.

\section{References}

1. Feng $X$ and McDonald JM (2011) Disorders of bone remodelling. Annu Rev Pathol 6: 121-145.

2. Weaver CM, Gordon CM, Janz KF, Kalkwarf HJ, Lappe JM, et al. (2016) The National Osteoporosis Foundation's position statement on peak bone mass development and lifestyle factors: asystematic review and implementation recommendations. Osteoporos Int 27(4): 1281-386

3. Cummings SR, Melton LJ (2002) Epidemiology and outcomes of osteoporotic fractures. Lancet 359: 1761-1777.

4. Johnell O, Kanis JA (2006) An estimate of the worldwide prevalence and disability associated with osteoporotic fractures. Osteoporos Int 17: 17261733.

5. Svedbom A, Hernlund E, Ivergard M, Cooper C, Stenmark J, et al. (2013) Osteoporosis in the European Union: A compendium of country-specific reports. Arch Osteoporos 8: 136.

6. Bruggmann D, Maule LS, Klingelhofer D, Schoffel N, Gerber A, et al (2016) World-wide architecture of osteoporosis research: density-equalizing mapping studies and gender analysis. Climacteric 19: 463-470.

7. Njeh CF, Boivin CM, Langton CM (1997) The role of ultrasound in the assessment of osteoporosis: A review. Osteoporos Int 7: 7-22.

8. Truscott JG (1997) Reference data for ultrasonic bone measurement: variation with age in 2087 Caucasian women aged 16-93 years. Brit J Radio 70: 1010-1016.

9. Roux C, Roberjot V, Porcher R, Kolta S, Dougados M, et al. (2001) Ultrasonic backscatter and transmission parameters at the oscalcis in postmenopausal osteoporosis. J Bone and Miner Res 16: 1353-1362.

10. Kanis JA, Melton LJ, Christiansen C, Johnston CC, Khaltaev N (1994) The diagnosis of osteoporosis. J Bone Miner Res 9: 1137-1141.

11. World Health Organization Study Group (1994) Assessment of fracture risk and its application to screening for postmenopausal osteoporosis. Report of a WHO Study Group. World Health Organ Tech Rep Ser 843: 1-129.

12. Clowes JA, Peel NFA, Eastell R (2006) Device-specific thresholds to diagnose osteoporosis at the proximal femur: an approach to interpreting peripheral bone measurements in clinical practice. Osteoporos Int 17: 1293-1302.

13. Kirchengast S (1993) Anthropometric-hormonal correlation patterns in fertile and post-menopausal women from Austria. Ann Hum Biol 20(1): 47-65.

14. Bedogni G, Mussi C, Malavolti M, Borghi A, Poli M, et al. (2002) Relationship between body composition and bone mineral content in young and elderly women. Ann Hum Biol 29: 559-565.

15. World Health Organization (2003) Prevention and management of osteoporosis: report of a WHO scientific group (No. 921). Diamond Pocket Books (P) Ltd, India.
16. Lane NE (2006) Epidemiology, etiology, and diagnosis of osteoporosis. Am J Obstet Gynecol 194: S3-S11.

17. Sioka C, Fotopoulos A, Georgiou A, Xourgia X, Papadopoulos A, et al. (2010) Age at menarche, age at menopause and duration of fertility as risk factors for osteoporosis. Climacteric 13(1): 63-71.

18. Compston JE, Flahive J, Hosmer DW, Watts NB, Siris ES, et al. (2014) Relationship of weight, height, and body mass index with fracture risk at different sites in postmenopausal women: the Global Longitudinal study of Osteoporosis in Women (GLOW). J Bone Miner Res 29: 487-493.

19. Zsakai A, Mascie-Taylor N, Bodzsar EB (2015) Relationship between some indicators of reproductive history, body fatness and the menopausal transition in Hungarian women. J PhysiolAnthrop 34(1): 1.

20. Drinkwater D, Ross W (1980) Anthropometric fractionation of body mass. Kinanthropometry II. University Park Press, Baltimore, USA

21. Greenspan SL, Bouxsein ML, Melton ME, Kolodny AH, Clair JH, et al (1997) Precision and discriminatory ability of calcaneal bone assessment technologies. J Bone Miner Res 12: 1303-1313.

22. Machado ABC, Hannon R, Henry Y, Eastell R (1997) Standardized coefficient of variation for dual energy $x$-ray absorptiometry (DXA), quantitative ultrasound (QUS) and markers of bone turnover. J Bone Miner Res 12: S258.

23. Morris R, Mazess R, Trempe J, Hanson J (1997) Stiffness compensates for temperature variation in ultrasound densitometry. J Bone Miner Res 12: S388.

24. Cole TJ, Green PJ (1992) Smoothing reference centile curves: The LMS method and penalized likelihood. Stat Med 11: 1305-1319.

25. Cole T, Pan H (2004) LMS program version 1.29. A program for calculating age-related reference centiles using the LMS method. Institute for Child Health, London, England.

26. Gluer CC, International Quantitative Ultrasound Consensus Group (1997) Quantitative ultrasound techniques for the assessment of osteoporosis: expert agreement on current status. J Bone Miner Res 12: 1280-1288.

27. Kim CH, Kim YI, Choi CS, Park JY, Lee MS, et al. (2000) Prevalence and risk factors of low quantitative ultrasound values of calcaneus in Korean elderly women. Ultrasound Med Biol 26: 35-40.

\section{Author Affiliations}

${ }^{1}$ Department of Biological Anthropology, Eotvos Lorand University, Pazmany P. s. 1/c, 1117 Budapest, Hungary

${ }^{2}$ Department of Biological Anthropology, University of Cambridge, Pembroke Street, Cambridge CB2 3RA, UK

Submit your next manuscript and get advantages of SciTechnol submissions

* 80 Journals

* 21 Day rapid review process

* 3000 Editorial tean

* 5 Million readers

- More than 5000 facebook

* Quality and quick review processing through Editorial Manager System

Submit your next manuscript at • www.scitechnol.com/submission 